\title{
Effectiveness and Safety of Golimumab in Patients with Ulcerative Colitis: A Multicenter, Prospective, Postmarketing Surveillance Study
}

\author{
Jongwook Yuㄹ, Soo Jung Park¹, Hyung Wook Kim², Yun Jeong Lim³ ${ }^{3}$, Jihye Park ${ }^{1,4}$, Jae Myung Cha ${ }^{5}$, Byong Duk \\ $\mathrm{Ye}^{6}$, Tae Oh Kim${ }^{7}$, Hyun-Soo Kim ${ }^{8}$, Hyun Seok Lee ${ }^{9,10}$, Su Young Jung ${ }^{11}$, Youngdoe Kim ${ }^{11}$, and Chang Hwan Choi ${ }^{12}$ \\ ${ }^{1}$ Department of Internal Medicine and Institute of Gastroenterology, Yonsei University College of Medicine, Seoul, ${ }^{2}$ Department of \\ Internal Medicine, Pusan National University Yangsan Hospital, Yangsan, ${ }^{3}$ Department of Internal Medicine, Dongguk University Ilsan \\ Hospital, Dongguk University College of Medicine, Goyang, ${ }^{4}$ Department of Internal Medicine, Seoul National University Bundang \\ Hospital, Seongnam, ${ }^{5}$ Department of Internal Medicine, Kyung Hee University School of Medicine, ${ }^{6}$ Department of Gastroenterology \\ and Inflammatory Bowel Disease Center, Asan Medical Center, University of Ulsan College of Medicine, Seoul, ${ }^{7}$ Department of \\ Internal Medicine, Inje University Haeundae Paik Hospital, Busan, ${ }^{8}$ Department of Internal Medicine, Yonsei University Wonju College \\ of Medicine, Wonju, ${ }^{9}$ Department of Internal Medicine, School of Medicine, Kyungpook National University, ${ }^{10} \mathrm{Kyungpook}$ National \\ University Hospital, Daegu, ${ }^{11}$ Medical Affairs, Janssen Korea Ltd., and ${ }^{12}$ Department of Internal Medicine, Chung-Ang University \\ College of Medicine, Seoul, Korea
}

\author{
Article Info \\ Received July 20, 2021 \\ Revised October 20, 2021 \\ Accepted October 21, 2021 \\ Published online December 27, 2021

\section{Corresponding Author \\ Chang Hwan Choi \\ ORCID https://orcid.org/0000-0001-7089-532X \\ E-mail gicch@cau.ac.kr}

Jongwook Yu and Soo Jung Park contributed equally to this work as first authors.
Background/Aims: Golimumab has been used for patients with ulcerative colitis (UC) since 2013. However, there is limited data on the effectiveness and safety of the real-world use of golimumab in Asian patients.

Methods: This was a multicenter, prospective, observational study. We enrolled patients with moderate-to-severe UC who were administered subcutaneous golimumab at 46 medical centers between May 2014 and November 2019. The primary outcome was the effectiveness and safety of golimumab at week 22. Clinical outcomes and adverse events were assessed according to partial Mayo score at weeks $0,2,6,14$, and 22.

Results: A total of 130 patients were included (mean age: $45.7 \pm 16.0$ years). The clinical response/ remission rates at weeks $2,6,14$, and 22 were $40.4 \% / 22.9 \%, 56.0 \% / 35.8 \%, 70.6 \% / 49.5 \%$, and $67.9 \% / 48.6 \%$, respectively. Based on full Mayo score at week 14 , clinical response and remission rates were $84.2 \%$ and $39.5 \%$, respectively. Mucosal healing rate was $65.8 \%$. In multivariate analysis with logistic regression, longer disease duration was significantly associated with a higher clinical response rate (adjusted odds ratio [aOR], 1.136; 95\% confidence interval [Cl], 1.006 to $1.282 ; p=0.040$ at week $6 ; a O R, 1.256 ; 95 \% \mathrm{Cl}, 1.049$ to $1.503 ; p=0.013$ at week 22). A higher baseline Mayo endoscopic subscore was significantly associated with a lower clinical response rate at week $6(a O R, 0.248 ; 95 \% \mathrm{Cl}, 0.089$ to $0.692 ; \mathrm{p}=0.008)$. The incidence of adverse drug reactions was $4.6 \%$ (6/130, nine events). No serious unexpected adverse drug reactions or deaths were reported.

Conclusions: Golimumab was effective and safe as an induction and maintenance treatment for Korean patients with moderate-to-severe UC. (Gut Liver 2022;16:764-774)

Key Words: Ulcerative colitis; Golimumab; Tumor necrosis factor- $\alpha$

\section{INTRODUCTION}

Ulcerative colitis (UC) is an inflammatory bowel disease that has a chronic relapsing-remitting disease course. The prevalence of UC has been increasing over the decades all over the world. Although the exact etiology has not been elucidated, immunologic, genetic, and environmental factors are known to be associated with the pathophysiology of UC. ${ }^{1-3}$

For the management of UC, the cornerstones of medi- 
cal treatment have been 5-aminosalicylic acids, various forms of corticosteroids, and immunomodulators such as azathioprine and 6-mercaptopurine. ${ }^{4-8}$ Since a few decades ago, anti-tumor necrosis factor $\alpha$ (anti-TNF- $\alpha$ ) agents have been used to control UC in patients who are refractory to conventional treatments. Many studies have proved the effectiveness and safety of anti-TNF- $\alpha$ agents in managing $\mathrm{UC}$, and thus they have become a valuable treatment option to spare patients from exposure to corticosteroids that are related to serious side effects. Recently, the timing of anti-TNF- $\alpha$ therapy has been getting earlier, and they are sometimes used very early for patients with acute severe UC. ${ }^{9,10}$

Besides infliximab, the commonly used biologic and small molecule agents for UC include adalimumab, golimumab, vedolizumab, ustekinumab, and tofacitinib. Recent studies have ascertained the effectiveness and safety of these new classes of medications in Asian patients. ${ }^{11-16}$ Their different biologic mechanisms and, most importantly, side effects are not well-known because of their relatively short period of time since development. ${ }^{16,17}$ Among them, golimumab belongs to a relatively new class of antiTNF- $\alpha$ agents (a fully human antibody to TNF- $\alpha$ ). It was investigated in the PURSUIT trial and has been prescribed for UC since 2013, although it had been used to manage various kinds of rheumatologic diseases. ${ }^{18-23}$ Golimumab has been proved effective in patients with UC, but there are not enough studies addressing its effect on patients who failed with conventional treatments such as 5-aminosalicylic acids, immunomodulators, steroids, and even other anti-TNF- $\alpha$ agents. ${ }^{24}$ Moreover, the effectiveness and safety of golimumab in patients with UC are mainly assessed in Western countries; its effectiveness and side effects for patients with UC in Asian countries are limited. ${ }^{25-28}$ Because golimumab has already been used in many Asian countries, it is necessary to evaluate its effectiveness and safety in a well-designed prospective study considering different drug responsiveness and disease characteristics among different ethnic groups. In this study, we prospectively evaluated the effectiveness and safety of golimumab in Korean patients with moderate-to-severe UC.

\section{MATERIALS AND METHODS}

\section{Study design}

This was a multicenter, prospective, postmarketing surveillance trial. We enrolled adult patients with moderately to severely active UC who have had an inadequate response or were intolerant to prior conventional treatments such as 5-aminosalicylic acids, corticosteroids, or immunomodu- lators. Golimumab $200 \mathrm{mg}$ subcutaneous (SC) injections were given on the first day of induction, followed by 100 mg SC injection 2 weeks later. Then, the patients received $100 \mathrm{mg}$ SC injection every 4 weeks. The primary objective was to evaluate the effectiveness and safety profiles in patients with UC who were administered with golimumab at week 22 . Effectiveness and safety were evaluated at weeks $2,6,14$, and 22 . The patients were analyzed for sex, age, height, weight, disease duration, Mayo score (full or partial if endoscopic results were not available), and concomitant medications to control UC at the time of enrollment. They were also investigated for adverse events (AEs) and adverse drug reactions (ADRs) during treatment with golimumab.

\section{Ethical considerations}

This study was performed in accordance with the ethical guidelines of the 1975 Declaration of Helsinki, and it was approved by the institutional review boards of all participating hospitals, including the Chung-Ang University Hospital (IRB number: C2016002). All patients agreed and signed their informed consents.

\section{Assessment of clinical outcomes}

In this study, clinical outcomes were assessed using Mayo scores (full or partial if endoscopic results were not available) measured by gastroenterologists at 46 medical centers in South Korea.

To assess the effectiveness of golimumab, partial Mayo (pMayo) score was measured at study entry and weeks 2, 6, 14, and 22. Effectiveness was evaluated in all patients who completed effectiveness assessments at baseline and at least once afterward. Clinical response was defined as a decrease from baseline in pMayo score by at least 2 points (full Mayo of at least 3 points) and at least $30 \%$ with an accompanying decrease in rectal bleeding subscore of at least 1 point or an absolute rectal bleeding subscore of 0 or 1 point. Clinical remission was defined as a pMayo score of 0 or 1 point (full Mayo score of 2 points or lower with a maximum of 1 in any of the subscores). Mucosal healing rate was defined as Mayo endoscopic subscore of 0 and 1. Last observation carried forward imputation methods were used when follow-up data were missed.

Corticosteroid-free clinical response and remission were defined when clinical response and remission were achieved without corticosteroids at the time points of weeks $6,14,22$ for the patients who did not receive corticosteroids during study period.

\section{Safety evaluation}

Safety was evaluated in patients who received golimumab at least once and underwent safety follow-up at least 
once. AEs were assessed by physical examination and routine questioning, with a follow-up of all patients to record all the AEs. The AEs were categorized by System Organ Class and Preferred Term using the terms provided in the Precautions for Use of golimumab and the Medical Dictionary for Regulatory Activities version 22.0. ${ }^{29}$ The causal relationship between an $\mathrm{AE}$ and golimumab was assessed by the investigator into six categories: certain, probable/ likely, possible, unlikely, conditional/unclassified, and unassessable/unclassifiable. In our study, all AEs not considered "unlikely" to be associated with golimumab were termed ADRs. Severity was assessed using the common categorical terms (mild, moderate, and severe). The ADRs not documented in the approved label information of golimumab was considered unexpected ADRs.

\section{Statistical analysis}

Descriptive statistics for continuous variables are presented as means with standard deviation or median (interquartile range) and dichotomous variables are presented as frequencies with percentages in parentheses. Patient characteristics, medical history, comorbidity, medication uses and AEs were summarized by descriptive statistics. We used, where appropriate, the Wilcoxon signed-rank test for change in pMayo score from baseline at each visit with missing data imputation with last observation carried forward method. In addition, multiple logistic regression analyses were used, as appropriate, to assess the adjusted effect of each clinical characteristic (sex, age at enrollment, age at diagnosis, disease duration, pMayo score at baseline, full Mayo score at baseline, Mayo endoscopy subscore at baseline (severe/moderate), prior biologic use, concomitant corticosteroid use, and concomitant immunomodulator use) on clinical response, remission and incidence of AEs. Analyses were performed using two-sided and p-values less than 0.05 were considered statistically significant. Analyses were performed using statistical software package SAS 9.4 (SAS Institute, Cary, NC, USA).

\section{RESULTS}

\section{Baseline patient characteristics}

A total of 133 patients with moderate-to-severe UC were prospectively enrolled from 46 teaching hospitals between May 23, 2014, and November 22, 2019. Among them, 130 patients were included in the safety analysis, while 109 patients were included in the effectiveness analysis. Seventy-one patients completed effectiveness assessments during regular visits for 22 weeks.

Of the 130 patients in total, $90(69.2 \%)$ were male and
$40(30.8 \%)$ were female, and their mean age was $45.7 \pm 16.0$ years. The mean age at the diagnosis of UC was $40.0 \pm 15.4$ years, and the median disease duration since diagnosis at the time of enrollment was 3.9 years (range, 1.5 to 8.0 years) (Table 1). Ninety-two patients (70.8\%) had colonoscopy or sigmoidoscopy at baseline, with the most common finding being moderate disease activity (Mayo endoscopic subscore 2 ) in 58 patients (63.0\%), followed by severe disease activity (Mayo endoscopic subscore 3) in 30 patients (32.6\%). The most common form of comorbidity was latent tuberculosis (TB), which was present in 14 patients (21.5\%), and all of them received standard treatment for latent TB before the first dose of golimumab. Although 41 patients (33.6\%) received systemic corticosteroids at baseline, 26 patients

Table 1. Baseline Patient Characteristics

\begin{tabular}{lc}
\hline \multicolumn{1}{c}{ Characteristics } & Value (n=130) \\
\hline Male sex & $90(69.2)$ \\
BMI, kg/m² & $22.3 \pm 2.9$ \\
Age at study enrollment, yr & $45.7 \pm 16.0$ \\
Age at diagnosis, yr & $40.0 \pm 15.4$ \\
Disease duration, yr* & $3.9(1.5-8.0)$ \\
Colonoscopy or sigmoidoscopy findings & $92(70.8)$ \\
Mild (Mayo endoscopic score 1) & $4(4.4)$ \\
Moderate (Mayo endoscopic score 2) & $58(63.0)$ \\
Severe (Mayo endoscopic score 3) & $30(32.6)$ \\
Full Mayo score (n=90) at study enrollment & $8(4-12)$ \\
Partial Mayo score (n=126) at study enrollment & $6(1-9)$ \\
Prior UC medications within 12 wk of study & $119(91.5)$ \\
enrollment ${ }^{\dagger}$ & \\
5-ASA & $114(95.8)$ \\
Systemic corticosteroids & $39(32.8)$ \\
Immunomodulators & $51(42.9)$ \\
Biologics & $18(15.1)$ \\
Concurrent UC medications used with & $122(93.9)$ \\
golimumab & \\
5-ASA & $117(95.9)$ \\
Systemic corticosteroids & $41(33.6)$ \\
Immunomodulators & $53(43.4)$ \\
Previous medical histories & $65(50.0)$ \\
Latent tuberculosis $_{\text {Pulmonary tuberculosis }}$ & $14(21.5)$ \\
Malignancies & $1(1.5)$ \\
Chronic hepatitis B & $5(7.5)$ \\
Others ${ }^{\ddagger}$ & $3(4.6)$ \\
\hline & $42(32.3)$ \\
\hline
\end{tabular}

Data are presented as number $(\%)$, mean \pm SD, or median (interquartile range).

BMI, body mass index; UC, ulcerative colitis; 5-ASA, 5-aminosalicylic acid.

*From the date of initial diagnosis to the date of first dose of subcutaneous golimumab; ${ }^{\dagger} 5$-ASA: mesalazine, balsalazide, sulfasalazine; Immunomodulators: azathioprine, mercaptopurine, methotrexate; Systemic steroids: prednisolone, methylprednisolone, dexamethasone, hydrocortisone; Biologics: infliximab, adalimumab, vedolizum$a b ;{ }^{\ddagger}$ Hypertension, diabetes mellitus, hyperlipidemia, coronary artery disease, psoriasis, etc. 
(63.4\%) could discontinue the systemic corticosteroids after treatment with golimumab within the median duration of 33 days (interquartile range, 15 to 45 days).

\section{Effectiveness of golimumab}

In our study, the retention probability of golimumab was $74.8 \%$ at week 22 (95\% confidence interval [CI], 67.5\% to $82.9 \%$ ) (Fig. 1). The study was prematurely discontinued in 49 patients (Table 2) due to lack of effectiveness (21 patients, $16.2 \%$ ), loss to follow-up (seven patients, 5.4\%), economic reason (one patient, $0.8 \%$ ), AEs (four patients, $3.1 \%$ ), and so on. The clinical response and remission rates were analyzed in 109 patients who completed effectiveness assessments at baseline and at least once afterward. The median pMayo score was 6 (range, 5 to 7 ) at baseline, 4 (range, 2 to 6) at week 2, 3 (range, 2 to 6) at week 6, 2 (range, 1 to 4 ) at week 14, and 2 (range, 1 to 4 ) at week 22. The pMayo score decreased significantly $(\mathrm{p}<0.001)$ from baseline at each follow-up point. The clinical response rates of the 109 patients at weeks 2, 6, 14, and 22 were $40.4 \%$ (95\% CI, $31.1 \%$ to $50.2 \%$ ), $56.0 \%$ (95\% CI, $46.1 \%$ to $65.5 \%$ ), $70.6 \%$ (95\% CI, $61.2 \%$ to $79.0 \%$ ), and $67.9 \%(95 \%$ CI, $58.3 \%$ to $76.5 \%$ ), respectively (Fig. 2 ). The clinical re- mission rates at weeks $2,6,14$, and 22 were $22.9 \%$ (95\% CI, $15.4 \%$ to $32.0 \%$ ), $35.8 \%$ (95\% CI, $26.8 \%$ to $45.5 \%$ ), $49.5 \%$ (95\% CI, $39.8 \%$ to $59.3 \%$ ), and $48.6 \%$ (95\% CI, $38.9 \%$ to 58.4\%), respectively (Fig. 2).

Full Mayo score was available in 38 patients (34.9\%) at week 14 (Fig. 3), with a median full Mayo score of 3 (interquartile range, 2 to 4). Mucosal healing at week 14 was achieved in 25 patients $(65.8 \% ; 95 \% \mathrm{CI}, 48.7 \%$ to

Table 2. Reasons for Study Discontinuation

\begin{tabular}{lc}
\hline \multicolumn{1}{c}{ Category* } & No. $(\%)(\mathrm{n}=130)$ \\
\hline Lack of effectiveness & $21(16.2)$ \\
Loss to follow-up & $7(5.4)$ \\
Adverse events & $4(3.1)$ \\
Sufficient effectiveness & $1(0.8)$ \\
Economic causes & $1(0.8)$ \\
Patient decision & $5(3.8)$ \\
Others & $10(7.7)$ \\
Termination of study & $9(6.9)$ \\
Investigator's leave & $1(0.8)$
\end{tabular}

*Category of the churn event: study discontinuation due to patients' decision, lack of effectiveness, economic cause, or adverse events; others were considered as censored data.

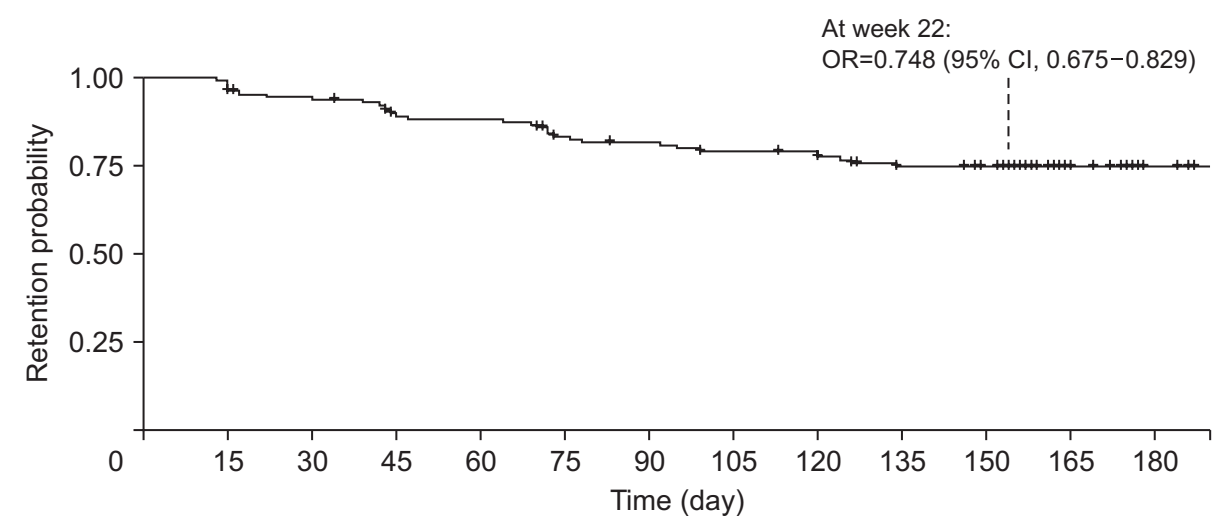

No. at risk

$\begin{array}{lllllllllllll}130 & 129 & 121 & 112 & 110 & 100 & 97 & 93 & 92 & 81 & 74 & 21 & 11\end{array}$

Fig. 1. Drug survival rate during the study.

$\mathrm{OR}$, odds ratio; $\mathrm{Cl}$, confidence inter-

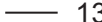

93
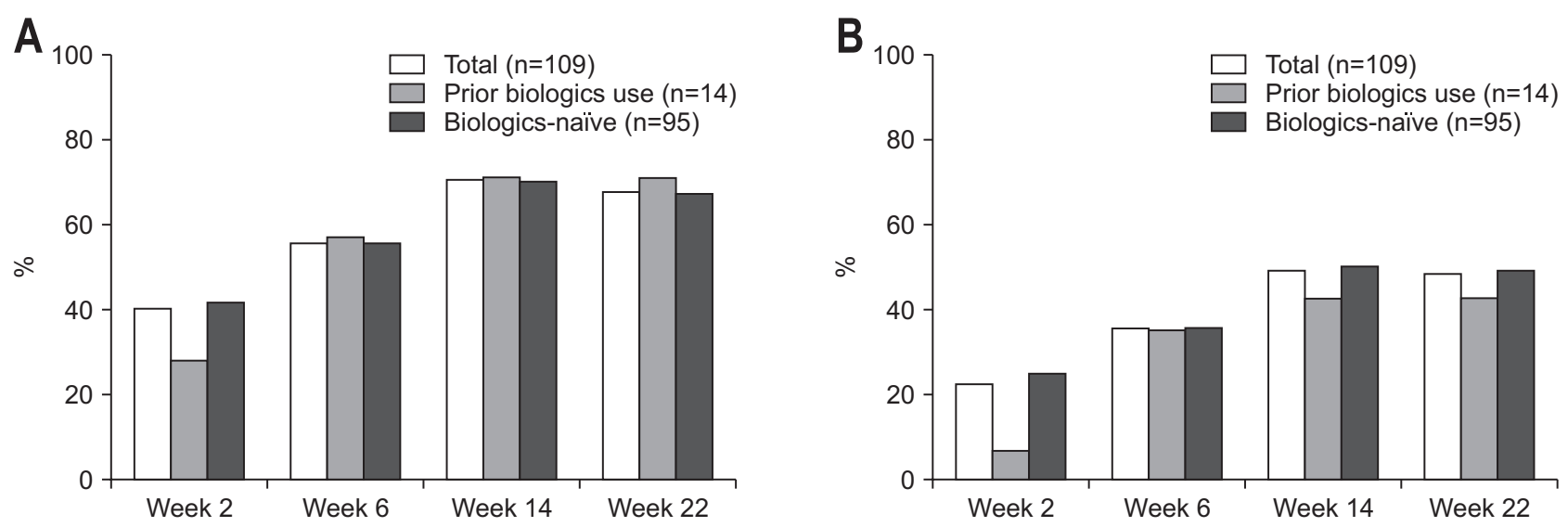

Fig. 2. Clinical response rate (A) and clinical remission rate (B) by visit. 
80.4\%). Endoscopic data was available in seven patients at week 22 with mucosa healing rate and complete mucosa healing rate of $71.4 \%(5 / 7)$ and $14.3 \%(1 / 7)$, respectively. Based only on the full Mayo score at week 14, the clinical response rate was $84.2 \%$ ( $95 \% \mathrm{CI}, 68.8 \%$ to $94.0 \%$ ), and the clinical remission rate was $39.5 \%$ (95\% CI, $24.0 \%$ to $56.6 \%)$ (Fig. 3).

Corticosteroid-free clinical response and remission rates in 74 patients who have not received corticosteroids

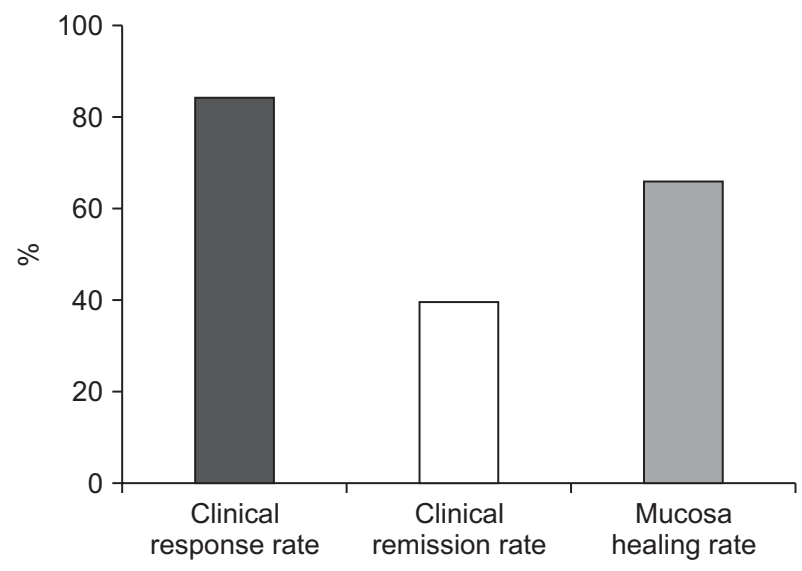

Fig. 3. Clinical outcomes by full Mayo score at week 14. during the study period were $55.4 \%, 35.1 \%$ at week 6 , $68.9 \%, 47.3 \%$ at week 14 , and $63.5 \%, 45.9 \%$ at week 22 , respectively.

\section{Clinical outcomes by univariable and multivariable Cox regression analyses}

On multivariate analysis with logistic regression for clinical response, longer disease duration was significantly associated with higher clinical response rate both at week 6 and week 22 (adjusted odds ratio [aOR], 1.136; 95\% CI, 1.006 to $1.282 ; \mathrm{p}=0.040$ at week 6 ; aOR, $1.256 ; 95 \% \mathrm{CI}$, 1.049 to $1.503 ; \mathrm{p}=0.013$ at week 22 , respectively) (Table 3 ). In addition, a higher baseline Mayo endoscopic subscore (severe degree to moderate degree) was associated with a significantly lower clinical response rate at week 6 (aOR, 0.248; 95\% CI, 0.089 to $0.692, \mathrm{p}=0.008$ at week 6) (Table 3). On multivariate analysis with logistic regression for clinical remission, a higher baseline Mayo endoscopic subscore (severe degree to moderate degree) was associated with a significantly lower clinical remission rate only at week 6 (aOR, 0.334; 95\% CI, 0.111 to $1.001 ; \mathrm{p}=0.050$ ) (Table 4). Fourteen out of the 109 patients had a history of prior biologics use (12.8\%). There was no significant relationship between prior biologics use and clinical response or remission rate in this study (Fig. 2).

Table 3. Logistic Regression Analysis for Clinical Response at Weeks 6 and 22

\begin{tabular}{|c|c|c|c|c|c|c|c|c|}
\hline \multirow{3}{*}{ Variable } & \multicolumn{4}{|c|}{ Week 6} & \multicolumn{4}{|c|}{ Week 22} \\
\hline & \multicolumn{2}{|c|}{ Univariable analysis } & \multicolumn{2}{|c|}{ Multiple analysis } & \multicolumn{2}{|c|}{ Univariable analysis } & \multicolumn{2}{|c|}{ Multiple analysis } \\
\hline & $\mathrm{OR}(95 \% \mathrm{Cl})$ & $\mathrm{p}$-value & OR $(95 \% \mathrm{Cl})$ & $\mathrm{p}$-value & OR $(95 \% \mathrm{Cl})$ & $p$-value & OR $(95 \% \mathrm{Cl})$ & $\mathrm{p}$-value \\
\hline Sex (female/male) & $\begin{array}{c}0.648 \\
(0.285-1.475)\end{array}$ & 0.301 & - & - & $\begin{array}{c}1.127 \\
(0.466-2.727)\end{array}$ & 0.790 & - & - \\
\hline $\begin{array}{l}\text { Age at study } \\
\text { enrollment }\end{array}$ & $\begin{array}{c}1.008 \\
(0.984-1.032)\end{array}$ & 0.534 & - & - & $\begin{array}{c}1.020 \\
(0.993-1.047)\end{array}$ & 0.144 & $\begin{array}{c}1.006 \\
(0.968-1.046)\end{array}$ & 0.756 \\
\hline Age at diagnosis & $\begin{array}{c}0.999 \\
(0.974-1.025)\end{array}$ & 0.959 & - & - & $\begin{array}{c}1.006 \\
(0.979-1.034)\end{array}$ & 0.657 & - & - \\
\hline Disease duration & $\begin{array}{c}1.079 \\
(0.997-1.168)\end{array}$ & 0.058 & $\begin{array}{c}1.136 \\
(1.006-1.282)\end{array}$ & 0.040 & $\begin{array}{c}1.176 \\
(1.048-1.320)\end{array}$ & 0.006 & $\begin{array}{c}1.256 \\
(1.049-1.503)\end{array}$ & 0.013 \\
\hline Prior biologics use & $\begin{array}{c}1.056 \\
(0.340-3.281)\end{array}$ & 0.924 & - & - & $\begin{array}{c}1.211 \\
(0.352-4.169)\end{array}$ & 0.762 & - & - \\
\hline Concomitant steroid & $\begin{array}{c}1.073 \\
(0.477-2.415)\end{array}$ & 0.865 & - & - & $\begin{array}{c}1.939 \\
(0.773-4.865)\end{array}$ & 0.158 & $\begin{array}{c}1.554 \\
(0.498-4.854)\end{array}$ & 0.448 \\
\hline $\begin{array}{l}\text { Concomitant } \\
\text { immunomodulator }\end{array}$ & $\begin{array}{c}1.134 \\
(0.525-2.448)\end{array}$ & 0.749 & - & - & $\begin{array}{c}1.289 \\
(0.565-2.944)\end{array}$ & 0.546 & - & - \\
\hline $\begin{array}{l}\text { Partial Mayo score at } \\
\text { baseline }\end{array}$ & $\begin{array}{c}1.162 \\
(0.889-1.519)\end{array}$ & 0.271 & - & - & $\begin{array}{c}1.170 \\
(0.884-1.549)\end{array}$ & 0.273 & - & - \\
\hline $\begin{array}{l}\text { Full Mayo score at } \\
\text { baseline }\end{array}$ & $\begin{array}{c}0.966 \\
(0.731-1.277)\end{array}$ & 0.811 & - & - & $\begin{array}{c}1.024 \\
(0.755-1.389)\end{array}$ & 0.879 & - & - \\
\hline $\begin{array}{l}\text { Mayo endoscopy score } \\
\text { at baseline (severe/ } \\
\text { moderate)* }\end{array}$ & $\begin{array}{c}0.279 \\
(0.105-0.739)\end{array}$ & 0.010 & $\begin{array}{c}0.248 \\
(0.089-0.692)\end{array}$ & 0.008 & $\begin{array}{c}0.510 \\
(0.185-1.404)\end{array}$ & 0.193 & $\begin{array}{c}0.426 \\
(0.141-1.294)\end{array}$ & 0.132 \\
\hline
\end{tabular}

$\mathrm{OR}$, odds ratio; $\mathrm{Cl}$, confidence interval.

*Due to the small number of subjects who had a mild Mayo endoscopy score at baseline, these subjects were excluded from the logistic regression; Variables with $p$-values $<0.2$ in univariable analysis were used for multivariate analysis. 
Table 4. Logistic Regression Analysis of Clinical Remission at Weeks 6 and 22

\begin{tabular}{|c|c|c|c|c|c|c|c|c|}
\hline \multirow{3}{*}{ Variable } & \multicolumn{4}{|c|}{ Week 6} & \multicolumn{4}{|c|}{ Week 22} \\
\hline & \multicolumn{2}{|c|}{ Univariable analysis } & \multicolumn{2}{|c|}{ Multiple analysis } & \multicolumn{2}{|c|}{ Univariable analysis } & \multicolumn{2}{|c|}{ Multiple analysis } \\
\hline & OR $(95 \% \mathrm{Cl})$ & $\mathrm{p}$-value & $\mathrm{OR}(95 \% \mathrm{CI})$ & $\mathrm{p}$-value & OR $(95 \% \mathrm{Cl})$ & $\mathrm{p}$-value & $\mathrm{OR}(95 \% \mathrm{Cl})$ & $\mathrm{p}$-value \\
\hline Sex (female/male) & $\begin{array}{c}1.037 \\
(0.443-2.428)\end{array}$ & 0.933 & - & - & $\begin{array}{c}1.676 \\
(0.735-3.826)\end{array}$ & 0.220 & - & - \\
\hline Age & $\begin{array}{c}1.013 \\
(0.987-1.038)\end{array}$ & 0.331 & - & - & $\begin{array}{c}1.016 \\
(0.992-1.041)\end{array}$ & 0.188 & $\begin{array}{c}1.008 \\
(0.976-1.040)\end{array}$ & 0.641 \\
\hline Age at diagnosis & $\begin{array}{c}1.006 \\
(0.980-1.033)\end{array}$ & 0.635 & - & - & $\begin{array}{c}1.004 \\
(0.979-1.030)\end{array}$ & 0.747 & - & - \\
\hline Disease duration & $\begin{array}{c}1.057 \\
(0.986-1.133)\end{array}$ & 0.116 & $\begin{array}{c}1.076 \\
(0.973-1.190)\end{array}$ & 0.151 & $\begin{array}{c}1.123 \\
(1.032-1.223)\end{array}$ & 0.007 & $\begin{array}{c}1.105 \\
(0.995-1.228)\end{array}$ & 0.063 \\
\hline Prior biologics use & $\begin{array}{c}0.997 \\
(0.309-3.214)\end{array}$ & 0.996 & - & - & $\begin{array}{c}0.766 \\
(0.247-2.377)\end{array}$ & 0.644 & - & - \\
\hline Concomitant steroid & $\begin{array}{c}1.091 \\
(0.473-2.515)\end{array}$ & 0.838 & - & - & $\begin{array}{c}1.397 \\
(0.623-3.131)\end{array}$ & 0.417 & - & - \\
\hline $\begin{array}{l}\text { Concomitant } \\
\text { immunomodulator }\end{array}$ & $\begin{array}{c}0.833 \\
(0.374-1.855)\end{array}$ & 0.655 & - & - & $\begin{array}{c}1.018 \\
(0.475-2.184)\end{array}$ & 0.963 & - & - \\
\hline $\begin{array}{l}\text { Partial Mayo score at } \\
\text { baseline }\end{array}$ & $\begin{array}{c}0.814 \\
(0.616-1.074)\end{array}$ & 0.146 & $\begin{array}{c}0.926 \\
(0.645-1.328)\end{array}$ & 0.675 & $\begin{array}{c}0.947 \\
(0.728-1.231)\end{array}$ & 0.684 & - & - \\
\hline $\begin{array}{l}\text { Full Mayo score at } \\
\text { baseline }\end{array}$ & $\begin{array}{c}0.759 \\
(0.562-1.024)\end{array}$ & 0.071 & - & - & $\begin{array}{c}0.942 \\
(0.716-1.239)\end{array}$ & 0.667 & - & - \\
\hline $\begin{array}{l}\text { Mayo endoscopy score } \\
\text { at baseline (severe/ } \\
\text { moderate)* }\end{array}$ & $\begin{array}{c}0.333 \\
(0.116-0.961)\end{array}$ & 0.042 & $\begin{array}{c}0.334 \\
(0.111-1.001)\end{array}$ & 0.050 & $\begin{array}{c}0.736 \\
(0.290-1.871)\end{array}$ & 0.520 & - & - \\
\hline
\end{tabular}

$\mathrm{OR}$, odds ratio; $\mathrm{Cl}$, confidence interval.

*Due to the small number of subjects who had a mild Mayo endoscopy score at baseline, these subjects were excluded from the logistic regression; Variables with $p$-values $<0.2$ in univariable analysis were used for multivariate analysis.

Table 5. Summary Incidence of Adverse Events and Adverse Drug Reactions

\begin{tabular}{lcc}
\multicolumn{1}{c}{ Summary } & No. of patients (\%) & No. of events \\
\hline No. of patients in the safety set & 130 & 43 \\
AEs & $28(21.5)$ & 13 \\
Most frequent AEs by organ system ( $\geq 2 \%$ of all patients) & $11(8.5)$ & 13 \\
Gastrointestinal disorders & $10(7.6)$ & 3 \\
Infections and infestations & $3(2.3)$ & 9 \\
Respiratory, thoracic, and mediastinal disorders & $6(4.6)$ & 2 \\
ADRs by preferred term & $2(1.5)$ & 1 \\
Anemia & $1(0.8)$ & 1 \\
Abdominal pain* & $1(0.8)$ & 1 \\
Allergic rhinitis* & $1(0.8)$ & 1 \\
Anal hemorrhage* & $1(0.8)$ & 1 \\
Palpitation* & $1(0.8)$ & 1 \\
Pneumonia & $1(0.8)$ & 1 \\
Pyrexia & $1(0.8)$ & 1 \\
Tuberculosis & & \\
\hline
\end{tabular}

AEs, adverse events; ADRs, adverse drug reactions.

*Unexpected AEs.

The patients who achieved clinical response or remission at week 6 were more likely to maintain clinical response or remission at week 14 and week 22 than the patients who could not achieve (for clinical response, $98.4 \%$ vs $35.4 \%$, $\mathrm{p}<0.001$ at week 14 and $90.2 \%$ vs $39.6 \%$, $\mathrm{p}<0.001$ at week 22 ; for clinical remission, $97.4 \%$ vs $22.9 \%$, p $<0.001$ at week 14 and $92.3 \%$ vs $24.3 \%, \mathrm{p}<0.001$ at week 22 ).

\section{Safety of golimumab}

Overall, 43 AEs were reported in 28 patients, with $21.5 \%$ of 130 patients experiencing at least one $\mathrm{AE}$ (Table 5). The most frequently reported AEs by System Organ Class were 
Table 6. Logistic Regression Analysis for Adverse Drug Events

\begin{tabular}{lcc}
\hline & Variable & Univariable analysis \\
\cline { 2 - 3 } & OR (95\% Cl) & $p$-value \\
\hline Sex (female/male) & $1.333(0.552-3.223)$ & 0.523 \\
Age & $1.013(0.987-1.040)$ & 0.333 \\
Age at diagnosis & $1.014(0.986-1.041)$ & 0.331 \\
Disease duration & $1.007(0.935-1.084)$ & 0.859 \\
Prior biologics use & $0.446(0.096-2.079)$ & 0.304 \\
Concomitant steroid & $1.553(0.651-3.706)$ & 0.321 \\
Concomitant immunomodulator & $1.116(0.479-2.602)$ & 0.780 \\
Partial Mayo score at baseline & $0.801(0.592-1.083)$ & 0.150 \\
Full Mayo score at baseline & $0.800(0.559-1.146)$ & 0.224 \\
Mayo endoscopy score at baseline (severe/moderate)* & $1.980(0.674-5.813)$ & 0.214 \\
\hline
\end{tabular}

$\mathrm{OR}$, odds ratio; $\mathrm{Cl}$, confidence interval.

*Due to the small number of subjects who had a mild Mayo endoscopy score at baseline, these subjects were excluded from the logistic regression; variables with $p$-values $<0.2$ in univariable analysis were used for multivariate analysis.

gastrointestinal disorders (8.5\%; 11/130 subjects, 13 events), followed by infections and infestations $(7.6 \% ; 10 / 130 \mathrm{pa}-$ tients, 13 events), and respiratory, thoracic, mediastinal disorders $(2.3 \% ; 3 / 130$ patients, three events). The incidence of ADRs was 4.6\% (6/130 patients, nine events; one event of mild abdominal pain, two events of mild anemia, one event of mild allergic rhinitis, one event of mild palpitation, one event of mild anal hemorrhage, one event of mild pyrexia, one event of mild TB, and one event of moderate pneumonia), with serious ADRs in $0.8 \%$ (1/130 patients, two events; pneumonia and TB) (Table 5). One patient aged 55 years developed active TB and permanently discontinued with golimumab treatment without recovery until study completion; this was evaluated as probable/likely association with golimumab as one of ADRs, but not as an unexpected event. All other eight ADRs were not required additional action on golimumab treatment. Serious unexpected ADRs and deaths were not reported during the study period. There were no significant factors associated with AEs (Table $6)$.

\section{DISCUSSION}

Since the introduction of biologics for the management of IBD, their role in the treatment of UC has steadily increased. Among anti-TNF- $\alpha$ agents, golimumab has been considered as one of the most common biologics to treat UC since 2013 due to the easily accessible administration route (SC) and relatively long injection intervals. As there have been few reports about the effectiveness and safety of golimumab in Asian patients, we followed 133 patients with moderate-to-severe UC of about 5 years in 46 teaching hospitals in South Korea to evaluate them in real world. ${ }^{30}$
Our study demonstrated the effectiveness of golimumab in Korean patients with moderate-to-severe UC that was consistent with previous studies. ${ }^{19,25,31,32}$ After induction dosage regimen of golimumab, more than half of the patients (56\%) achieved clinical response at week 6, and more than two-thirds of the patients (67.9\%) achieved clinical response at week 22. Of note, about half of the patients achieved clinical remission after week 14 (49.5\% at week 14 and $48.6 \%$ at week 22). Clinical response and remission rates were similar or even higher compared to other studies. ${ }^{19,25,31,32}$ Golimumab is known to reach peak serum concentrations in 2 to 6 days, obtaining steady drug levels after 14 weeks of treatment. ${ }^{25}$ In alignment with this result, clinical outcomes on full Mayo score were plateaued after week 14 , and the findings were consistent in other studies (clinical response rate, $84.2 \%$; mucosal healing rate, $65.8 \%$; clinical remission rate, 39.5\%) (Fig. 3). ${ }^{26,28,31,33,34}$

An unexpected observation was that longer disease duration was associated with better clinical response both at week 6 and week 22 (Table 3). The median duration of the disease since diagnosis was 3.9 years (interquartile range, 1.5 to 8.0 years), which is relatively shorter than previous studies. ${ }^{25,31}$ In the past, anti-TNF- $\alpha$ agents were not considered as initial treatment options but, recently, these agents were used earlier based on top-down approach. Golimumab appeared in the market after infliximab and adalimumab and relatively shorter disease duration in this study could be reflected in this real-world practice. Although shorter disease duration was known to be related with better clinical outcomes in previous studies, in this study, contrary results were found in multiple logistic regression model. In addition, disease duration was significantly related with clinical response but not with clinical remission in our study. This unexpected study finding might be possibly caused by other unrevealed factors 
such as previous treatment failures although detailed data could not be collected due to the limitation of real-world observational study. Dulai et al. ${ }^{35}$ also reported that longer disease duration was revealed as one of significant predictors of corticosteroid-free clinical remission, regardless of previous exposure with anti-TNF- $\alpha$. We need further welldesigned prospective studies with bias control to prove this interesting finding.

On the other hand, the endoscopic activity at baseline seems to be considered as clinical factor to affect clinical outcomes of golimumab; severe versus moderate endoscopic activity was significantly associated with a lower clinical response rate only at week 6 but not at week 22 (Tables 3 and 4). This result based on the baseline endoscopic Mayo subscore was similar to the previous study. ${ }^{28}$ For clinical remission, similarly, severe baseline endoscopic activity was associated with a poorer clinical remission rate only at week 6 . In other words, objective severe baseline endoscopic findings might predict a poor clinical response rate and a lower clinical remission rate only in short-term follow-up, not in mid-term follow-up. However, 6 weeks are not long enough to predict satisfactory effectiveness of golimumab especially in patients with severe endoscopic activity, which suggests more time is required to have maximal effectiveness of this drug. In addition, the patients with severe baseline endoscopic activity might show slower improvement of clinical symptoms assessed by pMayo score. We need more studies to prove the association between baseline endoscopic activity and prognosis. Of interest, the patients who achieved clinical response or remission at week 6 (early responder) persistently maintained higher clinical response and remission rates (more than $90 \%$ for both) at follow-up, consistent with previous studies. ${ }^{26,27}$

Although some studies found that golimumab was more effective in anti-TNF-naive patients, there was no significant difference between anti-TNF-naïve and antiTNF-experienced groups in our study. ${ }^{24}$ It might be caused from the limited number of patients with prior biologic use $(n=14)$ compared to the patients without biologic experience $(\mathrm{n}=95)$. Additionally, previous anti-TNF exposure was not significantly associated with golimumab persistence in a previous study $(\mathrm{n}=136$, median duration of 530 days, anti-TNF-naïve vs prior anti-TNF experience, $\mathrm{p}=0.71){ }^{36}$ We need further studies to elucidate the possible effects of prior biologic use on clinical outcomes when using additional biologic agents.

In our study, about $60 \%$ of patients could maintain clinical response without corticosteroids during the followup period. This steroid sparing effect of golimumab was similar to or higher than previous studies. ${ }^{25,31}$
As for safety evaluation, there were 15 patients (11.5\%) with a history of TB (latent TB in 14 patients, pulmonary $\mathrm{TB}$ in one patient) and five patients with a history of cancer. All 14 patients with a history of latent TB completed standard treatment for the latent ТB before initiating golimumab. Golimumab was discontinued only in one case because of active pulmonary TB that might be related to the side effects of golimumab. It is well known that antiTNF- $\alpha$ agents increase the risk of reactivation of TB. ${ }^{37-39}$ Out of the 133 patients including 15 patients with a history of latent or active TB in our study, only one patient developed TB reactivation during the follow-up. Although long-term follow-up data are needed, this finding is concordant with previous finding that active surveillance and treatment for latent TB are required during anti-TNF- $\alpha$ treatment. ${ }^{40}$ The patients with a history of cancer showed no cancer recurrence during this study period.

This study was a multicenter, prospective, observational study without a control group to which the effectiveness of SC golimumab could be compared. Although plateaued drug effect seems to be maintained after week 14, followup period of 22 weeks was not long enough to evaluate long-term drug effectiveness such as longevity, durability and safety issues such as severe infection, increased risk of cancer or TB. We set the time point of primary outcomes as "week 22" in consideration of both golimumab administration schedule and insurance issues in South Korea. Though longer-term follow-up data are necessary to evaluate the long-term effectiveness, safety, and adverse effects of maintenance treatment with golimumab, it is difficult to continue uncontrolled postmarketing surveillance study in real clinical practice. Because of potential selection bias derived from the prematurely discontinued patients $(n=49)$, caution should be exercised when interpreting the significant findings of this study. Additionally, in this observational study, considerable data might be lost because they were not collected mandatorily. For example, in endoscopic data collection, there might be missing data without central review. Furthermore, the extent of disease on endoscopy (Montreal classification) could not be assessed for the patients who had underwent endoscopy (full colonoscopy or sigmoidoscopy). Disease extent are known to affect prognosis and assessing disease extent would have helped estimate the effectiveness of golimumab. Lastly, serum and fecal inflammatory markers, such as C-reactive protein, erythrocyte sedimentation rate, and fecal calprotectin, were not examined. In spite of a few limitations, this study is still valuable in aspect of demonstrating the effectiveness and safety of golimumab in real-world clinical practice in South Korea for the first time. The findings in this study are consistent with previous randomized controlled trials 
and other observational studies. The study findings in this study might provide supportive information to accumulate real-world evidence of golimumab throughout the world.

In conclusion, golimumab showed its effectiveness and safety in both the induction and maintenance phases for Korean patients with moderate-to-severe UC. The early clinical outcomes were better in patients with less severe endoscopic activity at baseline.

\section{CONFLICTS OF INTEREST}

B.D.Y. has received a research grant from Celltrion and Pfizer Korea; consulting fees from Abbvie Korea, Celltrion, Chong Kun Dang Pharm., CJ Red BIO, Daewoong Pharma., Ferring Korea, Janssen Korea, Kangstem Biotech, Medtronic Korea, Pfizer Korea, Shire Korea, Takeda Korea, IQVIA, Cornerstones Health, and Takeda; speaking fees from Abbvie Korea, Celltrion, Ferring Korea, Janssen Korea, Pfizer Korea, Shire Korea, Takeda Korea, Takeda and IQVIA. Y.K. and S.Y.J. are full-time employees of Janssen Korea. No other potential conflicts of interest relevant to this article were reported.

\section{ACKNOWLEDGEMENTS}

This study was funded and supported by Janssen Korea Ltd.

\section{AUTHOR CONTRIBUTIONS}

Conceptualization: S.J.P., C.H.C. Data collection and analysis: J.Y., S.J.P., H.W.K., Y.J.L., J.P., J.M.C., B.D.Y., T.O.K., H.S.K., H.S.L., Y.K., C.H.C. Statistical analysis: Y.K., S.Y.J. Analyzing and approval of manuscript: J.Y., S.J.P., C.H.C. Preparation the manuscript for the final submission: J.Y., S.J.P., C.H.C. All authors read and approved the final manuscript.

\section{ORCID}

Jongwook Yu https://orcid.org/0000-0001-7489-2222 Soo Jung Park https://orcid.org/0000-0003-0699-6809 Hyung Wook Kim https://orcid.org/0000-0002-2444-0591 Yun Jeong Lim https://orcid.org/0000-0002-3279-332X Jihye Park https://orcid.org/0000-0001-5253-2138 Jae Myung Cha https://orcid.org/0000-0001-9403-230X Byong Duk Ye https://orcid.org/0000-0001-6647-6325
Tae Oh Kim https://orcid.org/0000-0002-7359-1599 Hyun-Soo Kim https://orcid.org/0000-0001-7190-0362 Hyun Seok Lee https://orcid.org/0000-0001-9288-0321 Su Young Jung https://orcid.org/0000-0002-3946-1520 Youngdoe Kim https://orcid.org/0000-0002-0772-6360 Chang Hwan Choi https://orcid.org/0000-0001-7089-532X

\section{REFERENCES}

1. Sands BE. From symptom to diagnosis: clinical distinctions among various forms of intestinal inflammation. Gastroenterology 2004;126:1518-1532.

2. Jess T, Riis L, Vind I, et al. Changes in clinical characteristics, course, and prognosis of inflammatory bowel disease during the last 5 decades: a population-based study from Copenhagen, Denmark. Inflamm Bowel Dis 2007;13:481-489.

3. da Silva BC, Lyra AC, Rocha R, Santana GO. Epidemiology, demographic characteristics and prognostic predictors of ulcerative colitis. World J Gastroenterol 2014;20:9458-9467.

4. Meucci G, Fasoli R, Saibeni S, et al. Prognostic significance of endoscopic remission in patients with active ulcerative colitis treated with oral and topical mesalazine: a prospective, multicenter study. Inflamm Bowel Dis 2012;18:10061010 .

5. Triantafillidis JK, Merikas E, Georgopoulos F. Current and emerging drugs for the treatment of inflammatory bowel disease. Drug Des Devel Ther 2011;5:185-210.

6. Colombel JF, D'haens G, Lee WJ, Petersson J, Panaccione R. Outcomes and strategies to support a treat-to-target approach in inflammatory bowel disease: a systematic review. J Crohns Colitis 2020;14:254-266.

7. Ooi CJ, Hilmi I, Banerjee R, et al. Best practices on immunomodulators and biologic agents for ulcerative colitis and Crohn's disease in Asia. Intest Res 2019;17:285-310.

8. Singh A, Mahajan R, Kedia S, et al. Use of thiopurines in inflammatory bowel disease: an update. Intest Res 2022;20:1130 .

9. Oh SJ, Shin GY, Soh H, et al. Long-term outcomes of infliximab in a real-world multicenter cohort of patients with acute severe ulcerative colitis. Intest Res 2021;19:323-331.

10. Mahajan R, Singh A, Kedia S, et al. Maintaining infliximab induced clinical remission with azathioprine and 5-aminosalicylates in acute severe steroid-refractory ulcerative colitis has lower cost and high efficacy (MIRACLE): a multicenter study. Intest Res 2022;20:64-71.

11. Ogata H, Hagiwara T, Kawaberi T, Kobayashi M, Hibi T. Safety and effectiveness of adalimumab in the treatment of ulcerative colitis: results from a large-scale, prospective, multicenter, observational study. Intest Res 2021;19:419-429.

12. Shin SY, Park SJ, Kim Y, et al. Clinical outcomes and predic- 
tors of response for adalimumab in patients with moderately to severely active ulcerative colitis: a KASID prospective multicenter cohort study. Intest Res 2022;20:350-360.

13. Kobayashi T, Ito $\mathrm{H}$, Ashida $\mathrm{T}$, et al. Efficacy and safety of a new vedolizumab subcutaneous formulation in Japanese patients with moderately to severely active ulcerative colitis. Intest Res 2021;19:448-460.

14. Hisamatsu T, Kim HJ, Motoya S, et al. Efficacy and safety of ustekinumab in East Asian patients with moderately to severely active ulcerative colitis: a subpopulation analysis of global phase 3 induction and maintenance studies (UNIFI). Intest res 2021;19:386-397.

15. Oh EH, Kim J, Ham N, et al. Long-term outcomes of adalimumab therapy in Korean patients with ulcerative colitis: a hospital-based cohort study. Gut Liver 2020;14:347-356.

16. Lee YI, Park Y, Park SJ, Kim TI, Kim WH, Cheon JH. Comparison of long-term outcomes of infliximab versus adalimumab treatment in biologic-naïve patients with ulcerative colitis. Gut Liver 2021;15:232-242.

17. Barberio B, Savarino EV, Card T, et al. Incidence comparison of adverse events in patients with inflammatory bowel disease receiving different biologic agents: retrospective longterm evaluation. Intest Res 2022;20:114-123.

18. Harzallah I, Rigaill J, Williet N, Paul S, Roblin X. Golimumab pharmacokinetics in ulcerative colitis: a literature review. Therap Adv Gastroenterol 2017;10:89-100.

19. Sandborn WJ, Feagan BG, Marano C, et al. Subcutaneous golimumab induces clinical response and remission in patients with moderate-to-severe ulcerative colitis. Gastroenterology 2014;146:85-95.

20. Sandborn WJ, Feagan BG, Marano C, et al. Subcutaneous golimumab maintains clinical response in patients with moderate-to-severe ulcerative colitis. Gastroenterology 2014; 146:96-109.

21. Rutgeerts P, Feagan BG, Marano CW, et al. Randomised clinical trial: a placebo-controlled study of intravenous golimumab induction therapy for ulcerative colitis. Aliment Pharmacol Ther 2015;42:504-514.

22. Gibson PR, Feagan BG, Sandborn WJ, et al. Maintenance of efficacy and continuing safety of golimumab for active ulcerative colitis: PURSUIT-SC maintenance study extension through 1 year. Clin Transl Gastroenterol 2016;7:e168.

23. Adedokun OJ, Xu Z, Marano CW, et al. Pharmacokinetics and exposure-response relationship of golimumab in patients with moderately-to-severely active ulcerative colitis: results from phase $2 / 3$ PURSUIT induction and maintenance studies. J Crohns Colitis 2017;11:35-46.

24. Taxonera C, Rodríguez C, Bertoletti F, et al. clinical outcomes of golimumab as first, second or third anti-TNF agent in patients with moderate-to-severe ulcerative colitis. Inflamm Bowel Dis 2017;23:1394-1402.
25. Bosca-Watts MM, Cortes X, Iborra M, et al. Short-term effectiveness of golimumab for ulcerative colitis: observational multicenter study. World J Gastroenterol 2016;22:1043210439.

26. Hibi T, Imai Y, Senoo A, Ohta K, Ukyo Y. Efficacy and safety of golimumab 52-week maintenance therapy in Japanese patients with moderate to severely active ulcerative colitis: a phase 3, double-blind, randomized, placebo-controlled study-(PURSUIT-J study). J Gastroenterol 2017;52:11011111.

27. Reinisch W, Gibson PR, Sandborn WJ, et al. Long-term benefit of golimumab for patients with moderately to severely active ulcerative colitis: results from the PURSUIT-maintenance extension. J Crohns Colitis 2018;12:1053-1066.

28. Detrez I, Dreesen E, Van Stappen T, et al. Variability in golimumab exposure: a 'real-life' observational study in active ulcerative colitis. J Crohns Colitis 2016;10:575-581.

29. Brown EG, Wood L, Wood S. The medical dictionary for regulatory activities (MedDRA). Drug Saf 1999;20:109-117.

30. Hibi T, Kamae I, Pinton P, et al. Efficacy of biologic therapies for biologic-naïve Japanese patients with moderately to severely active ulcerative colitis: a network meta-analysis. Intest Res 2021;19:53-61.

31. Tursi A, Allegretta L, Della Valle N, et al. Effectiveness of golimumab in inducing remission and clinical response in outpatient ulcerative colitis. Clin Res Hepatol Gastroenterol 2016;40:e61-e63.

32. Olivera P, Danese S, Pouillon L, Bonovas S, Peyrin-Biroulet L. Effectiveness of golimumab in ulcerative colitis: a review of the real world evidence. Dig Liver Dis 2019;51:327-334.

33. Bossuyt P, Baert F, D'Heygere F, et al. Early mucosal healing predicts favorable outcomes in patients with moderate to severe ulcerative colitis treated with golimumab: data from the Real-life BE-SMART cohort. Inflamm Bowel Dis 2019;25:156-162.

34. Bossa F, Biscaglia G, Valvano MR, et al. Real-life effectiveness and safety of golimumab and its predictors of response in patients with ulcerative colitis. Dig Dis Sci 2020;65:17671776.

35. Dulai PS, Singh S, Casteele NV, et al. Development and validation of clinical scoring tool to predict outcomes of treatment with vedolizumab in patients with ulcerative colitis. Clin Gastroenterol Hepatol 2020;18:2952-2961.

36. Bressler B, Williamson M, Sattin B, Camacho F, Steinhart $\mathrm{AH}$. Real world effectiveness of golimumab therapy in ulcerative colitis regardless of prior TNF exposure. J Can Assoc Gastroenterol 2018;1:129-134.

37. Gardam MA, Keystone EC, Menzies R, et al. Anti-tumour necrosis factor agents and tuberculosis risk: mechanisms of action and clinical management. Lancet Infect Dis 2003;3: 148-155. 
38. Keane J. TNF-blocking agents and tuberculosis: new drugs illuminate an old topic. Rheumatology (Oxford) 2005;44:714-720.

39. Theis VS, Rhodes JM. Review article: minimizing tuberculosis during anti-tumour necrosis factor-alpha treatment of inflammatory bowel disease. Aliment Pharmacol Ther
2008;27:19-30.

40. Hsia EC, Cush JJ, Matteson EL, et al. Comprehensive tuberculosis screening program in patients with inflammatory arthritides treated with golimumab, a human anti-tumor necrosis factor antibody, in Phase III clinical trials. Arthritis Care Res (Hoboken) 2013;65:309-313. 